\title{
Effect of Pycnogenol on Skin Wound Healing
}

\author{
Moon-Jin Jeong, Soon-Jeong Jeong, Soo-Han Lee ${ }^{1}$, Young-Soo Kim ${ }^{2}$, Baik-Dong Choi, \\ Seung-Hyun Kim ${ }^{2}$, Ara Go ${ }^{2}$, Se Eun Kim ${ }^{2}$, Seong-Soo Kang ${ }^{2}$, Chang-Jong Moon ${ }^{2}$, \\ Jong-Choon $\mathrm{Kim}^{2}$, Sung-Ho Kim ${ }^{2}$, Chun-Sik Bae ${ }^{2, *}$
}

\author{
Department of Oral Histology and Developmental Biology, School of Dentistry, Chosun University, Gwangju 501-825, Korea \\ ${ }^{1}$ College of Veterinary Medicine, Konkuk University, Seoul 143-701, Korea \\ ${ }^{2}$ College of Veterinary Medicine, Chonnam National University, Gwangju 500-757, Korea
}

*Correspondence to:

Bae CS,

Tel: $+82-62-530-2876$

Fax: +82-62-530-2809

E-mail: csbae210@chonnam.ac.kr

Received October 11, 2013

Revised October 29, 2013

Accepted October 29, 2013
This study was carried out to investigate the effects of pycnogenol (PYC) on the cutaneous wound healing of the mice. The wounds were extracted on days 1, 3, 5, and 7 post-injury for histomorphometrical analysis including wound area, infiltrating inflammatory cells, wound contracture including collagen deposition. As the result, the wound area of PYCtreated group was larger than the control group on days 1 to 7 . Inflammatory cells in the PYC-treated wounds were decreased at day 1 compared to the control wound tissue. From day 3 to 7, there was no significant difference between the control and the PYC-treated skin wounds. Though the degree of contraction in the PYC-treated group was lower than that of the control group from days 1 to 5, but appeared significantly higher on day 7 . Compared to the control group, collagen accumulation in the PYC-treated group was higher than that of the control group from days 5 to 7. From this result, it may support the possibility that PYC would be useful agent for early inflammatory response and matrix remodeling phase of the skin wounds.

Key Words: Skin wound healing, Pycnogenol, Mice

\section{INTRODUCTION}

The wound healing is a complex process that can be divided into inflammatory reaction, proliferation and maturation of newly formed tissue. The inflammatory phase involves vascular and cellular events and is best characterized by edema, erythema and marked increase of blood supply. During proliferative phase there is formation of the epithelium with concomitant grow of granulation tissue and angiogenesis. Once the tissue within the wound is formed the maturation phase begins. The synthesis of collagens and other extracellular matrix components increase tensile strength of the wound (Chodorowska \& Roguś-Skorupska, 2004).

Pycnogenol (PYC), an aqueous extract of the bark of Pinus pinaster (formerly known as Plantago maritima), is primarily composed of procyanidins and phenolic acids. Procyanidins are biopolymers of catechin and epicatechin subunits which are recognized as important constituents in human nutrition. PYC contains a wide variety of procyanidins that range from the monomeric catechin and taxifolin to oligomers with 7 or more flavonoid subunits. The phenolic acids are derivatives of benzoic and cinnamic acids (Rohdewald, 2002). PYC protects against oxidative stress in several cell systems by doubling the intracellular synthesis of anti-oxidative enzymes and by acting as a potent scavenger of free radicals (Berryman et al., 2004). PYC has multiple biological effects, including antioxidant, anti-inflammatory and anticarcinogenic properties (Sime \& Reeve, 2004). Protection against ultraviolet (UV)-radiationinduced erythema was found in a clinical study following oral intake of PYC (Blazso et al., 1997). PYC can inhibit the expression of the proinflammatory cytokine interleukin-1 by regulating redox-sensitive transcription factors (Cho et al.,

This study was financially supported by Chonnam National University, 2011.

@ This is an open-access article distributed under the terms of the Creative Commons Attribution Non-Commercial License (http://creativecommons.org/licenses/by-nc/3.0) which permits unrestricted noncommercial use, distribution, and reproduction in any medium, provided the original work is properly cited.

Copyrights $(2013$ by Korean Society of Microscopy 
2000), and significantly shortened the wound healing time when applied topically to experimental wounds inflicted on healthy rats by means of a branding iron (Blazso et al., 2004). However, the recovery pattern of skin wounds caused by burns varies markedly from that caused by physical injury or surgery (Wang et al., 2008). PYC has been shown to be effective in the wound healing process; however, information on surgical skin wounds is little to lacking. Thus, this study was aimed at investigating the effects of PYC during the healing process at surgically induced skin wounds using histochemical and histomorphometric analysis.

\section{MATERIALS AND METHODS}

\section{Animals}

Male ICR mice $(n=24)$ were purchased from Samtako (Osan, Korea) and used after one week of quarantine and acclimatization. The animals were housed in polycarbonate cages in a room maintained at a temperature of $23^{\circ} \mathrm{C} \pm 2^{\circ} \mathrm{C}$, a relative humidity of 50\% $\pm 5 \%$, artificial lighting from 08:00 to 20:00 hour, and 13 to 18 air changes per hour. The animals were blindly randomized into control $(n=12)$ and PYC $(n=12)$ groups.

\section{Animal Model of the Incised Skin Wound}

A total of 24 male 6-week-old ICR mice were anesthetized by intraperitoneal injection of xylazine $\left(2 \mathrm{mg} / \mathrm{kg}\right.$, Rompun ${ }^{\circledR}$; Bayer Korea, Seoul, Korea) and zolazepam/tiletamine (0.1 mg/ kg, Zoletil 50 ${ }^{\circledR}$; Virbac Korea, Seoul, Korea). A scalpel was used to make four $1 \mathrm{~cm}$ long full-thickness incisions deep to the fascia on the central dorsum of each mouse. Specimens $(1.5 \times 2$ $\mathrm{cm}^{2}$ ) surrounding the wound was excised after the animal was euthanized by cervical dislocation while anesthetized on days $1,3,5$, and 7 post-incision ( $\mathrm{n}=3$ /group). The Institutional Animal Care and Use Committee of Chonnam National University approved the protocols for the animal study, and the animals were cared for in accordance with the Guidelines for Animal Experiments of Chonnam National University (CNU IACUC-YB-R-2012-10).

\section{Pycnogenol}

PYC was provided by the Horphag Research Ltd. (Route de Belis, France). The PYC ( $1 \mathrm{mg} / 50 \mu \mathrm{L} /$ wound $)$ was dissolved in saline and administered once subcutaneously prior to making the surgical skin wound in the treated group.

\section{Histological Analysis}

To compare changes between groups, mice were euthanized

A

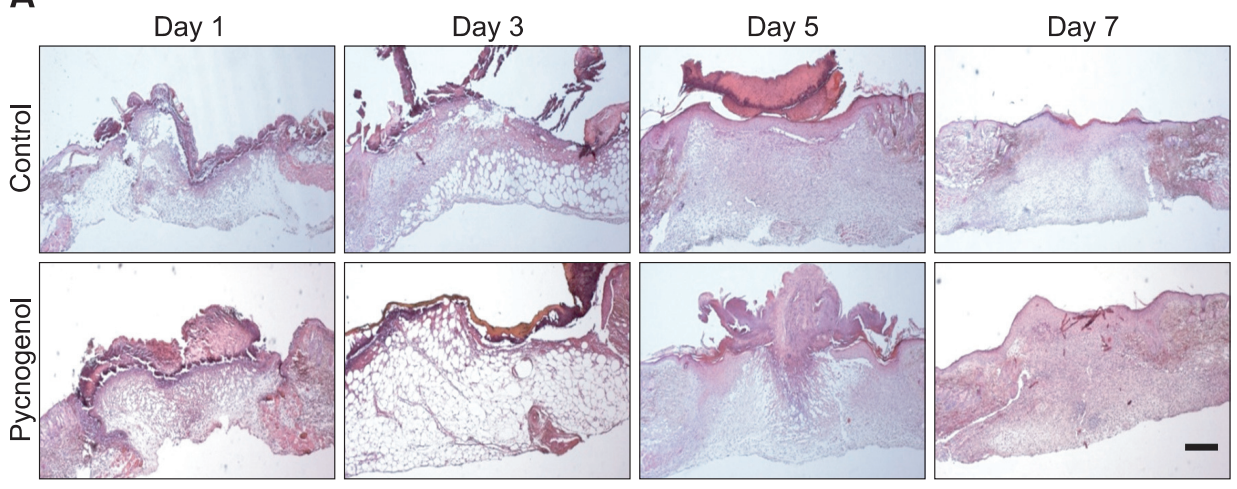

B

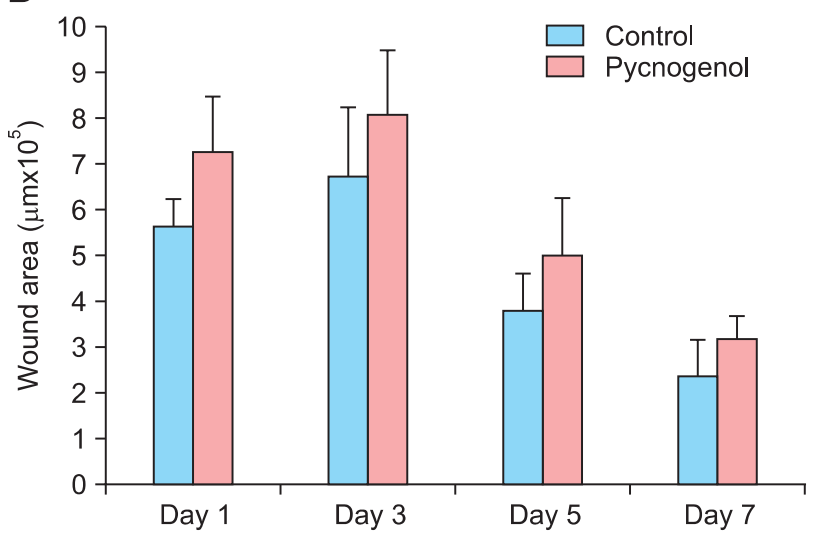

Fig. 1. (A) Differences in the wound area between the control and the pycnogenol (PYC)-treated groups during skin wound healing. There were no differences between the groups until day 3 . However, scar tissues were observed in the PYC-treated group on day 5 . H\&E stain, scale bar $=200$ $\mu \mathrm{m}$. (B) The wound area of the PYC-treated group was larger than the control group on days 1 to 7 , based upon morphometric analysis of the wounds tissues from the control and the PYC-treated groups. 
on days 1, 3, 5, and 7 post-wounding. Skin samples from the wound area were taken and $\mathrm{H} \& \mathrm{E}$ staining was performed to measure the size of the wound area and detect any histomorphometric changes. To analyze the number of inflammatory cells, Giemsa staining was performed, and to analyze the collagen in the matrix formation, Masson's trichrome and picrosirius red staining were used. The wound area was measured as well as the epithelium and the marginal area of the connective tissue. The number of inflammatory cells was counted at the left, right, and center of the wound area. Wound contraction was calculated by measuring the distance between the edge of the wound and the damaged dermal layer. Stained tissues were observed using a multiple microscope (Carl Zeiss, Oberkochen, Germany), and the AxioVision LE release 4.6 (Carl Zeiss) image analysis program was used for all analysis.

\section{Statistical Analysis}

Data presented are mean values from three animal \pm standard deviations. Statistical analysis of the data was performed using a Student's t-test. Differences were considered significant at $\mathrm{p}<0.01$.

\section{RESULTS}

\section{Histological and Morphometric Analysis of the Skin Wound Healing following Pycnogenol Treatment}

Most wound tissues of the control group appeared to be collapsed and lost on day 1, and scabs caused by blood clots and fibrous tissues were observed. The movement of epithelial cells was confirmed at day 5 and most dermal residues disappeared. On day 7 the wound site including the derma was markedly reduced compared to day 1 . The PYCtreated group showed a pattern similar to the control group until day 3 after the wound induction, but scabs were not removed until day 5 and movement of epithelial cells was also observed to be lower than that of the control group (Fig. 1A). The wound measurements showed that the wound area of the control group increased slightly on day 3 compared to day 1 , albeit not in a significant manner, and it decreased on day 5 . On day 7, the wound area decreased significantly. In the PYCtreated group, the wound size decreased from days 1 to 7 , this pattern was similar to that of the control (Fig. 1B).
A
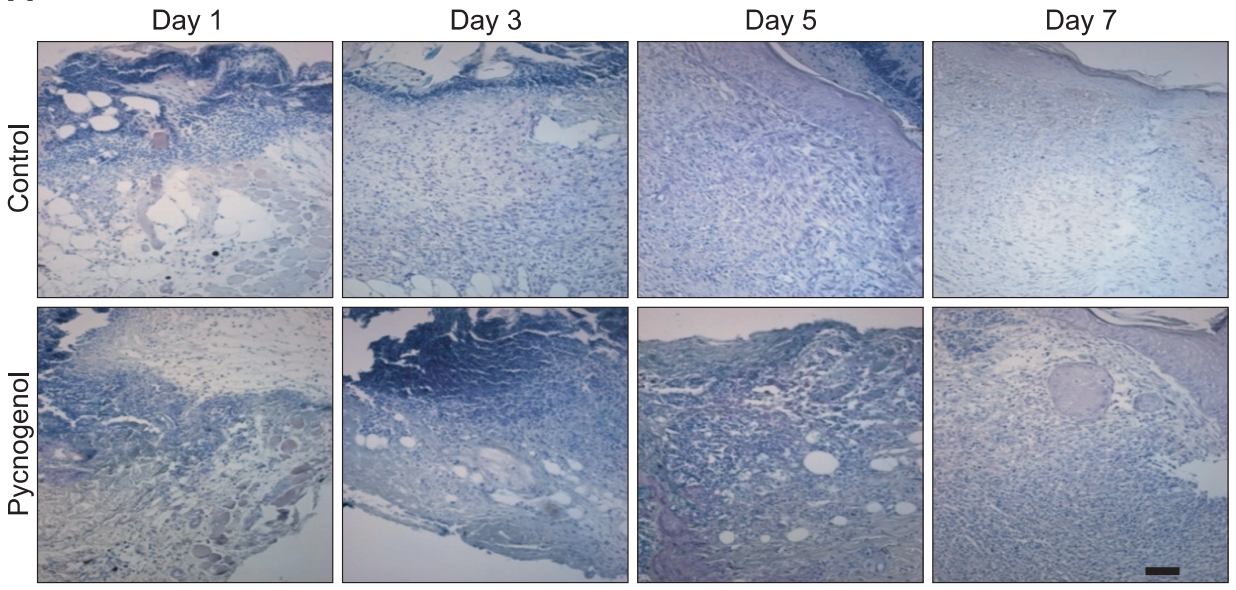

B

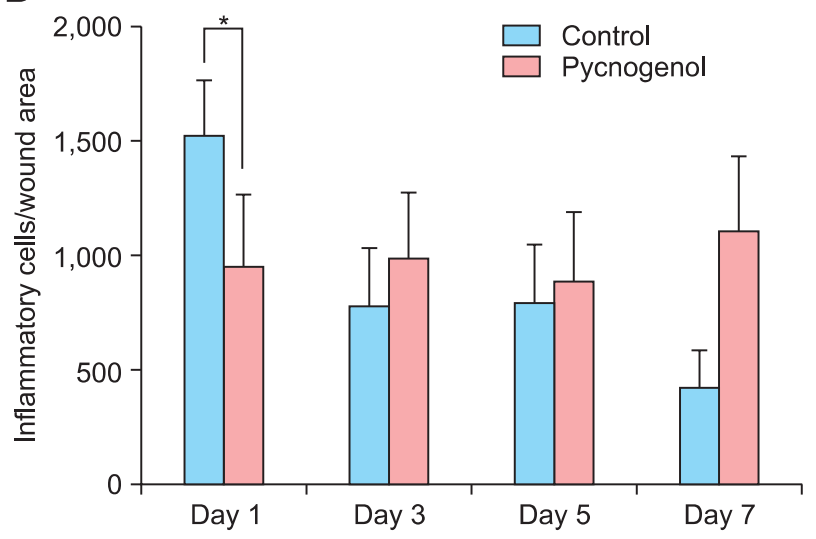

Fig. 2. (A) Differences in the recruitment of inflammatory cells between the control and the pycnogenol (PYC)-treated skin wounds. Inflammatory cells in the PYCtreated wounds were decreased at day 1 compared to the control wound tissue. From day 3 to 7 , there was no significant difference between the control and the PYC-treated skin wounds. All tissues were subjected to Giemsa staining. Scale bar $=20$ $\mu \mathrm{m}$. (B) Inflammatory cells were counted in three distinctive areas (left, right, and middle of the wound area). The number of inflammatory cells in the PYC-treated group was lower than the control group on day $1 .{ }^{*} \mathrm{p}<0.01$. 


\section{Histological Analysis of Inflammatory Cells during the Skin Wound Healing Process}

To compare the number of inflammatory cells recruited in the wound site, tissues that caused wounds were subjected to Giemsa staining (Fig. 2A), which showed the highest number of inflammatory cells in the control group on day 1 . But after day 3 , up to day 7 , the number declined sharply. It could be observed that in the PYC-treated group, there were less inflammatory cells than in the control group only on day 1 , but it maintained more inflammatory cells similar with the control group from days 3 to 7 (Fig. 2B).

A

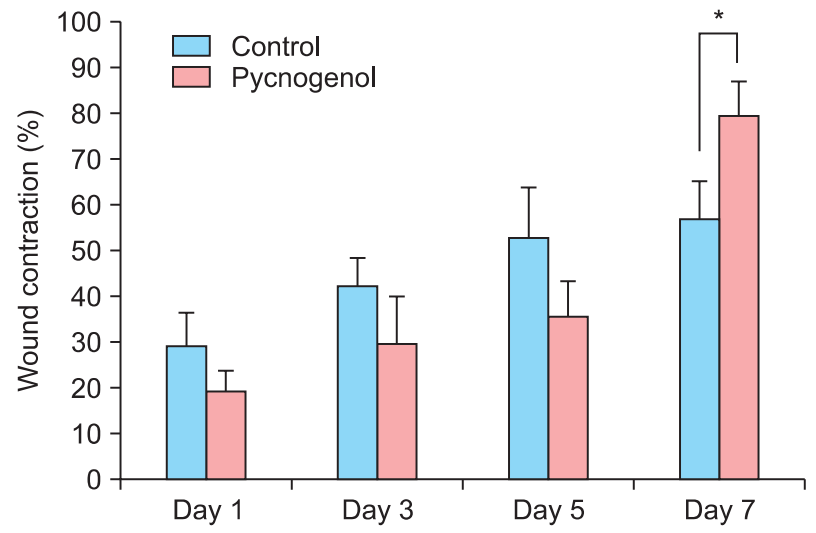

\section{Collagen Deposition and Remodeling in the Wound Healing Process}

Both the control and the PYC-treated groups showed an increasing tendency in the analysis of wound contraction. Though the degree of contraction in the PYC-treated group was lower than that of the control group from days 1 to 5 , but appeared higher on day 7 (Fig. 3A). To identify the collagen deposition Masson's trichrom staining and picrosirius red staining were conducted. In the control group, stained as light blue, was identified around the wound from around day 3 after inducing wounds. In the control and the PYC-treated groups, dark blue staining was observed in the tissues on

B
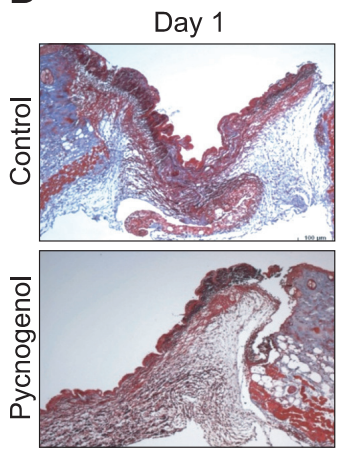

C

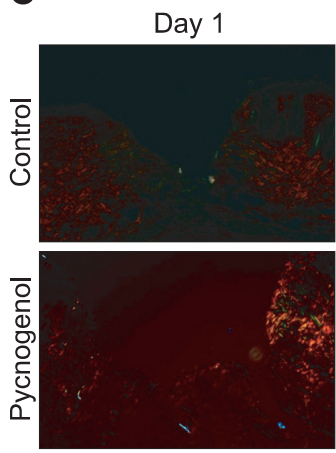

Day 3

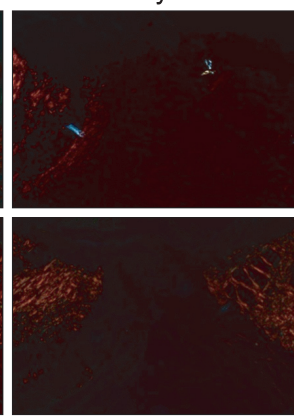

Day 5
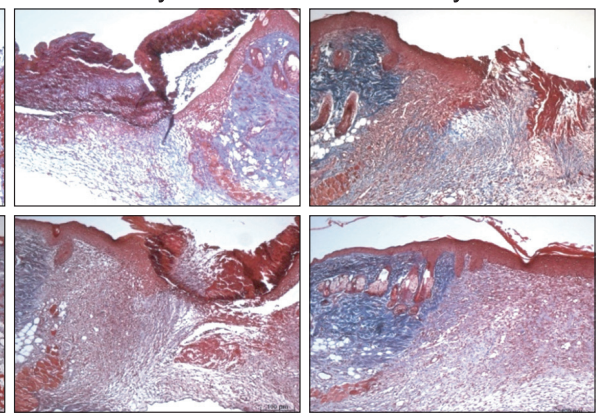

Day 5

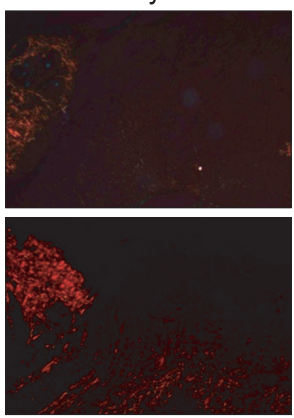

Day 7

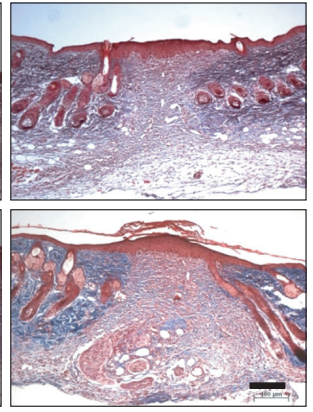

Day 7

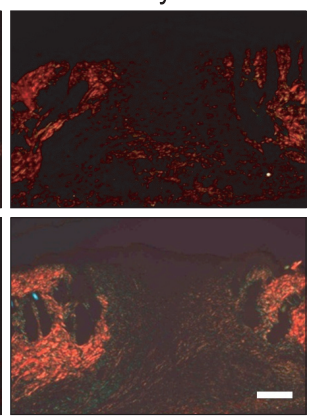

Fig. 3. (A) From day 1 to 5 , there was no significant difference between the control and the pycnogenol (PYC)-treated skin wounds. However, wound contraction in the PYC-treated group was significantly higher than the control group on day 7. ${ }^{*} \mathrm{p}<0.01$. (B) Difference in collagen accumulation (blue color) in the healing areas between the control and the PYCtreated groups. Collagen in the healing area of the control and the PYC-treated groups gradually increased from days 1 to 7. Collagen deposition in the PYC-treated group was greater than in the control group on days 5 and 7. Masson's trichrome stain, scale bar $=100 \mu \mathrm{m}$. (C) Difference in the accumulated collagen (bright red color) in the healing areas of the control and the PYC-treated groups. This result was consistent with the collagen staining. Picrosirius red stain, scale bar $=100 \mu \mathrm{m}$. 
days 5 and 7 (Fig. 3B). Quantitative collagen deposit analysis showed clear collagen synthesis from day 5 and increased on day 7. Compared to the control group, collagen accumulation in the PYC-treated group was higher than that of the control group from days 5 to 7 (Fig. 3C).

\section{DISCUSSION}

It is well established that skin wound healing is a complex but spatially and temporally controlled biological response which is generally divided into inflammatory, proliferative and tissue remodeling phase (Martin, 1997; Singer \& Clark, 1999). During the inflammatory phase, platelet aggregation at the injury site is followed by the infiltration of leukocytes such as neutrophils, macrophages, and T-lymphocytes into the wound site. In the proliferative phase, re-epithelialization and newly formed granulation tissue begin to cover the wound area to complete tissue repair. Cell biological studies demonstrated that many cytokines, growth factors, proteases, and so on are closely involved in the wound healing process to complete normal tissue repair after damage (Martin, 1997; Singer \& Clark, 1999). Major functions of neutrophils include phagocytosis, which eliminates the infective agents at the site of damaged tissue, and the stimulation of blood coagulation, inflammation, and the healing process by secreting various factors (Eming et al., 2007). Next, monocytes arrive at the wound within two days and differentiate into macrophage. They also function as phagocytes and antigen presenting cells, secreting transforming growth factors- and, basic fibroblast growth factor, and platelet-derived growth factor to regulate the wound healing process (Eming et al., 2007).

Re-epithelialization and granulation tissue formation occur simultaneously within a few hours of the inflammatory reaction. Keratinocytes that exist around the edges of the wound and in the residues of skin appendages begin to migrate into the wound and form a scab (Singer \& Clark, 1999). Almost simultaneously, as fibroblasts located around the undamaged dermis begin to proliferate and migrate upon stimulation by growth factors, granulation tissue formation is initiated (Singer \& Clark, 1999). Fibroblasts that migrate into the wound and proliferate synthesize proteoglycans, collagen type III, and collagen type I to reform the extracellular matrix around the wound (Clark, 1996). Some fibroblasts differentiate into myofibroblasts and synthesize smooth muscle actin, which provides mechanical tension to pull the edges of the wound to stimulate contraction and eventually promote wound closure (Hinz et al., 2001). The dermis remodeling phase of skin wound healing involves the full growth of the wound accompanied by collagenous scarring. The residual fibroblasts rearrange the collagen fiber to recover the original tension of the skin, repeating collagen deposition and degradation for several months. However, the original skin tension before the damage can never be fully recovered (Singer \& Clark, 1999; Hinz, 2007).

There are various medications available to assist in the skin wound healing and from the ancient times, various natural substances have been widely used for wound healing (Kim et al., 2011). Among such extracts, polyphenol compounds are known to be strong antioxidants capable of neutralizing free radicals by combining with active oxygen (Hsu, 2005). A hydroxyl group attaches to an aromatic ring of a polyphenol compound that combines with free radicals, which appear during the metabolic process and form neutralized stable phenoxyl radicals (Rice-Evans et al., 1996). All of these have been known to play a significant role not only in the biological environment but also in the treatment of diseases (Packer et al., 1999).

Radicals produced by wounds are largely superoxide radical anions produced by neutrophils and macrophages and also play an important role in the removal of microorganisms and pathogens (Babior, 2000). PYC, which is one of the polyphenol compounds, is a pine bark extract and has been used as a medicine for wound recovery and inflammatory diseases since the ancient times (Packer et al., 1999). Through basic research and clinical studies, PYC was known not only to be a powerful antioxidant (Chida et al., 1999; Devaraj et al., 2002) but as an agent to improve vascular integrity and endothelial function and to stimulate anti-inflammatory responses (Grimm et al., 2006). Also, PYC regulates nitric oxide production of activated macrophages and inhibits nitric oxide synthase (iNOS) activity and inducible macrophagetype NOS mRNA expression (Virgili et al., 1998). Based on the abundant evidence, this study aimed to identify the effect of PYC on wound recovery inducing skin incisions on the dermal layer by histological analysis.

Observing the speed of wound recovery with the naked eye showed no difference between the control group and the PYC-treated groups (data not shown). Also, the wound measurement was wider than that of the control group from days 1 to 7 after inducing wounds. The rates of keratinocyte migration were similar in both groups on day 1 but lower in the PYC-treated group than the control group thereafter (data not shown). This shows that treatment with PYC has no effect on the speed of wound recovery, which is not consistent with previous reports. PYC was demonstrated to promote wound recovery time in mice with treatment with 5\% PYC after induction of burn wounds was about three days less than the control group, and the measurement of the scar tissue was also 2.6 times less than the control group (Blazso et al., 2004). We speculate that the discrepancy may be due to a few reasons. First, the previous study used a higher concentration of PYC, $1 \%, 2 \%$, and $5 \%$, compared to our study. Second, there was difference in the absorption rate of PYC because the administration of PYC was in conjunction with a gel 
substance called "carbomer $934 \mathrm{P}$ " in the other studies. In our study, however, PYC was injected in the form of an aqueous solution. Methods to induce wounds in wound recovery experiments can be largely divided into burning, full thickness incision, and excision of some tissues. In most other studies, the most common form of wound induction was burning, unlike this current study. Grimm et al. (2006) reported that PYC promotes inflammatory responses, in that PYC interferes with matrix metalloproteinase- 9 secretion by monocytes. Also, PYC has been shown to inhibit the inflammatory response of UV-induced wounds in a concentration-dependent manner (Sime \& Reeve, 2004). When we examined the influx of inflammatory cells, there were less inflammatory cells in the PYC-treated group than in the control group only on day 1 . From days 3 to 5, however, there were more inflammatory cells in this group than the control group. On day 7, a higher tendency to increase was observed in the treated group compared to the control group. Therefore, it appears that PYC inhibits inflammatory responses in the early period of wound healing, but as time passes, its effect wanes. However, both the degree of contraction of wounds and the collagen deposit time were higher in the PYC-treated group than the control group on day 7. PYC also stimulates the formation of collagen at the second half of the wound recovery period. Recently, one study showed that an Ocimum sanctum extract, a kind of polyphenol, increases the synthesis of collagen, which is the main ingredient, by promoting proliferation of fibroblasts (Shetty et al., 2007). Also, grape seed extracts, another powerful antioxidant, was demonstrated to stimulate the crosslinking of collagen produced in the fibroblasts (Han et al., 2003). Altogether, PYC appears to inhibit the inflammatory response during the wound recovery process in the early stage and to promote collagen deposition in the wound area.

\section{CONCLUSIONS}

When we investigated the effects of PYC during the healing process at surgically induced skin wounds using histochemical and histomorphometric analysis, PYC inhibited the initial inflammatory response and promoted collagen deposition and remodeling, suggesting that PYC would be useful agent for early inflammatory response and matrix remodeling phase of the skin wounds.

\section{REFERENCES}

Babior B M (2000) Phagocytes and oxidative stress. Am. J. Med. 109, 33 44.

Berryman A M, Maritim A C, Sanders R A, and Watkins III J B (2004) Influence of treatment of diabetic rats with combinations of pycnogenol, beta-carotene, and alpha-lipoic acid on parameters of oxidative stress. J. Biochem. Mol. Toxicol. 18, 345-352.

Blazso G, Gabor M, and Rohdewald P (1997) Anti-inflammatory activities of procyanidin containing extracts from Pinus pinaster Ait. after oral and cutaneous application. Pharmazie 52, 380-382.

Blazso G, Gabor M, Schonlau F, and Rohdewald P (2004) Pycnogenol accelerates wound healing and reduces scar formation. Phytother. Res. 18, 579-581.

Chida M, Suzuki K, Nakanishi-Ueda T, Ueda T, Koide R, and Armstrong D (1999) In vitro testing of antioxidants and biochemical end-point in bovine retinal tissue. Ophthalmic Res. 31, 407-415.

Cho K J, Yun C H, Yoon D Y, Cho Y S, Rimbach G, and Chung A S (2000) Effect of bioflavonoids extracted from the bark of Pinus maritima on proinflammatory cytokine interleukin-1 production in lipopolysaccharide- stimulated RAW 264.7. Toxicol. Appl. Pharmacol. 168, 64-71.

Chodorowska G and Roguś-Skorupska D (2004) Cutaneous wound healing. Ann. Univ. Mariae. Curie. Sklodowska. Med. 59, 403-407.

Clark R A F (1996) Wound repair: overview and general considerations. In: The Molecular and Cellular Biology of Wound Repair, eds. Clark R A and Henson P M, pp. 513-560, (Plenum Press, New York).

Devaraj S, Vega-López S, Kaul N, and Jialal I (2002) Supplementation with a pine bark extract rich in polyphenols increases plasma antioxidant capacity and alters the plasma lipoprotein profile. Lipids
37, 931-934.

Eming S A, Krieg T, and Davidson J M (2007) Inflammation in wound repair: molecular and cellular mechanisms. J. Invest. Dermatol. 127, 514-525.

Grimm T, Chovanová Z, Muchová J, Duracková Z, and Högger P (2006) Inhibition of NF-kappaB activation and MMP-9 secretion by plasma of human volunteers after ingestion of maritime pine bark extract (Pycnogenol). J. Inflamm. (Lond) 27, 1-6.

Han B, Jaurequi J, Tang B W, and Nimni M E (2003) Proanthocyanidin: a natural crosslinking reagent for stabilizing collagen matrices. J. Biomed. Mater. Res. A 65, 118-124.

Hinz B (2007) Formation and function of the myofibroblast during tissue repair. J. Invest. Dermatol. 127, 526-537.

Hinz B, Mastrangelo D, Iselin C E, and Gabbiani G (2001) Mechanical tension controls granulation tissue contractile activity and myofibroblast differentiation. Am. J. Pathol. 159, 1009-1020.

Hsu S (2005) Green tea and the skin. J. Am. Acad. Dermatol. 52, 10491059.

Kim Y S, Cho I H, Jeong M J, Jeong S J, Nah S Y, Cho Y S, Kim S H, Go A, Kim S E, Kang S S, Moon C J, Kim J C, Kim S H, and Bae C S (2011) Therapeutic effect of total ginseng saponin on skin wound healing. $J$. Ginseng Res. 35, 360-367.

Martin P (1997) Wound healing-aiming for perfect skin regeneration. Science 276, 75-81.

Packer L, Rimbach G, and Virgili F (1999) Antioxidant activity and biologic properties of a procyanidin-rich extract from pine (Pinus maritima) bark, pycnogenol. Free Radic. Biol. Med. 27, 704-724. 
Rice-Evans C A, Miller N J, and Paganga G (1996) Structure-antioxidant activity relationships of flavonoids and phenolic acids. Free Radic. Biol. Med. 20, 933-956.

Rohdewald P (2002) A review of the French maritime pine bark extract (Pycnogenol), a herbal medication with a diverse clinical pharmacology. Int. J. Clin. Pharmacol. Ther. 40, 158-168.

Shetty S, Udupa S, and Udupa L (2007) Evaluation of antioxidant and wound healing effects of alcoholic and aqueous extract of Ocimum sanctum Linn in rats. Evid. Based Complement Alternat. Med. 5 95-101.

Sime S and Reeve V E (2004) Protection from inflammation, immuno- suppression and carcinogenesis induced by UV radiation in mice by topical Pycnogenol. Photochem. Photobiol. 79, 193-198.

Singer A J and Clark R A (1999) Cutaneous wound healing. N. Engl. J. Med. 341, 738-746.

Virgili F, Kobuchi H, and Packer L (1998) Procyanidins extracted from Pinus maritima (Pycnogenol): scavengers of free radical species and modulators of nitrogen monoxide metabolism in activated murine RAW 264.7 macrophages. Free Radic. Biol. Med. 24, 1120-1129.

Wang Z Y, Zhang J, and Lu S L (2008) Objective evaluation of burn and post-surgical scars and the accuracy of subjective scar type judgment. Chin. Med. J. (Engl) 121, 2517-2520. 